\title{
Representability of Cones in Weighted Lebesgue Spaces and Extrapolation Operators on Cones
}

\author{
E. I. Berezhnoi* and L. Maligranda** \\ Presented by Academician S.M. Nikol'skii March 30, 2005
}

Received September 23, 2005

\section{DOI: $10.1134 / \mathrm{S} 1064562406010169$}

The role played by exact estimates of classical operators in harmonic analysis and adjacent areas is well known. In recent years, because of new problems of analysis, estimates of operators on some cones in spaces rather than on the entire spaces have become very popular (see, e.g., [1-4]). On the other hand, in the theory of integral operators with positive kernels, the extrapolation theorem of Schur (see, e.g., [5]) is well known; it says that an integral operator $K x(t)=$ $\int k(t, s) x(s) d s$ with $k(t, s) \geq 0$ is bounded in $L^{p}$ if and only if there exists a positive function $u(t)$ such that it is finite almost everywhere and the operator is bounded in the pairs $K: L_{u}^{\infty} \rightarrow L_{u}^{\infty}$ and $K: L_{v}^{1} \rightarrow L_{v}^{1}$, where $v=$ $u^{1 / p-1}$. In relation to various problems of analysis, the interest in extrapolation theorems has increased [6-8]. For this reason, it is natural to pass from the Lebesgue space $L^{p}$ to cones of Lebesgue spaces in these theorems.

In this paper, we suggest a reduction of estimating operators on cones to estimating them on new spaces, which are constructed from the cones and the initial spaces, for the most important cones in the Lebesgue spaces. Such a reduction makes it possible to apply the whole apparatus developed for obtaining exact estimates on weight Lebesgue spaces to obtain exact estimates of operators on cones. Using the reduction, we prove a new extrapolation theorem for a certain class of operators defined on cones in Lebesgue spaces.

Let $S(\mu)=S\left(R_{+}, \Sigma, \mu\right)$ [where $R_{+}=(0,+\infty)$ ] be the space of measurable functions $x: R_{+} \rightarrow R$. Recall that a Banach space $X=(X, \| \cdot|X|)$ consisting of measurable functions is said to be ideal [9] if, for any $y \in X$ and any measurable $x$ such that $|x(t)| \leq|y(t)|$ almost everywhere on $R_{+}, x \in X$ and $\left\|x|X|\left|\leq\|y \mid X\|\right.\right.$. As usual, the symbol $L^{p}$

\footnotetext{
* Yaroslavl State University, Sovetskaya ul. 14, Yaroslavl, 150000 Russia

** Luleå University of Technology, Luleå, S-97187 Sweden
}

(where $1 \leq p \leq \infty$ ) denotes the classical Lebesgue space.

Let $w: R_{+} \rightarrow R_{+}$be a positive function (weight). For an ideal space $X$, we use $X_{w}$ to denote the new ideal space with norm $\left\|x\left|X_{w}\|=\| w x\right| X\right\|$.

Definition 1. Let $X$ be an ideal space in $S(\mu)$, and let $K$ be a cone in $S(\mu)$. As usual, $K \cap X$ denotes the intersection of the cone $K$ with the cone $X_{+}$.

Let $K(\downarrow)$ denote the cone in $S(\mu)$ consisting of the functions $x: R_{+} \rightarrow R_{+}$that do not increase, i.e., satisfy the condition $x(t+h) \leq x(t)$ for $h \geq 0$, and let $K(\uparrow)$ be the cone of nondecreasing functions in $S(\mu)$; by $K(\downarrow, \uparrow)$ we denote the cone in $S(\mu)$ consisting of the concave functions $x: R_{+} \rightarrow R_{+}$satisfying the additional conditions $\lim _{t \rightarrow 0} x(t)=0$ and $\lim _{t \rightarrow 0} t^{-1} x(t)=0$.

Theorem 1. Suppose that $p \in(1, \infty)$ and $w$ is a weight function such that

$$
\int_{1}^{\infty} w^{p}(s) d s=\infty
$$

and

$$
\int_{0}^{t} w^{p}(s) d s<\infty \text { for any } t>0
$$

Let $Q$ be the operator defined by

$$
Q x(t)=\int_{t}^{\infty} x(\tau) d \tau .
$$

Finally, let $\mathrm{v}$ be a new function for which

$$
\left\|\kappa(0, t) w\left|L^{p}\|\cdot\| \kappa(t, \infty) \frac{1}{V}\right| L^{p^{\prime}}\right\| \equiv 1
$$

$[\kappa(D)$ denotes the characteristic function of the set $D]$. 
Then, the following assertions are valid: the operator $Q$ acts and is bounded in the pair

$$
Q:\left(L_{V}^{p}\right)_{+} \rightarrow K(\downarrow) \cap L_{w}^{p} ;
$$

there exists a constant $c>0$ such that, for any $y \in K(\downarrow) \cap$ $L_{w}^{p}$ with $\left\|y \mid L_{p}^{w}\right\|=1$, there exists a function $x \in L_{V}^{p}$ with $\left\|x \mid L_{\mathrm{V}}^{p}\right\|=1$ such that

$$
(Q x)(t) \geq c y(t)
$$

for any $t \in(0, \infty)$.

Note that condition (4) holds by virtue of the classical estimates of the operator $Q$ in the spaces $L_{u}^{p}$ [the weight $v$ in (3) was chosen so as to ensure this]; see, e.g., $[4,10]$. For $y \in K(\downarrow) \cap L_{w}^{p}$, the function $x$ in (5) is defined constructively.

Remark 1. Theorem 1 has a complete analogue for the cones $K(\uparrow), K(\varphi, \downarrow)=\left\{x: R_{\downarrow} \rightarrow R_{+}: \varphi(t) x(t) \downarrow\right\}$, and $K(\varphi, \uparrow)=\left\{x: R_{+} \rightarrow R_{+}: \varphi(t) x(t) \uparrow\right\}$ with the only difference that, instead of $Q$, the operators

$$
\begin{gathered}
P x(t)=\int_{0}^{t} x(s) d s, \quad Q_{\varphi} x(t)=\frac{1}{\varphi(t)} \int_{t}^{\infty} x(s) d s, \\
P_{\varphi} x(t)=\frac{1}{\varphi(t)} \int_{0}^{t} x(s) d s
\end{gathered}
$$

should be considered.

Let us exemplify the applications of Theorem 1.

Theorem 2. Suppose that $1 \leq p_{0}<p_{1}<\infty$ and $u$ and $w$ are weight functions satisfying conditions (1) and (2).

Then,

$$
K(\downarrow) \cap L_{u}^{p_{0}} \neq K(\downarrow) \cap L_{u}^{p_{1}} ;
$$

i.e., these cones do not coincide for any weight functions satisfying the assumptions of the theorem.

We say that an operator $T: S(\mu) \rightarrow S(\mu)$ is sublinear if $|T(x+y)(t)| \leq T|x|(t)+T|y|(t)$ and $|T(\lambda x)(t)| \leq \lambda|T x(t)|$ for $\lambda \geq 0$.

Theorem 1 immediately implies the following assertion.

Theorem 3. Suppose that $p \in(1, \infty)$ and $w$ is a weight function satisfying conditions (1) and (2). Let $Y$ be an ideal Banach space in $S(\mu)$.

A sublinear operator $T$ acts and is bounded as an operator from $K(\downarrow) \cap L_{w}^{p}$ to $Y$ if and only if the superposition operator $T Q$ acts and is bounded as an operator from $L_{v}^{p}$ to $Y$.

Using the technique for estimating operators $L: L_{w}^{p} \rightarrow$ $Y$ (see, e.g., $[2,4,11,12]$ ), we can obtain various esti- mates for operators on the cone of monotone functions in Lebesgue spaces by applying Theorem 3.

Consider the cone $K(\downarrow, \uparrow)$. For nonnegative functions on $R_{+}$, we define the operators

$$
Q_{1} x(t)=t \int_{t}^{\infty} x(s) d s \text { and } P_{1} x(t)=\int_{0}^{t} s x(s) d s .
$$

It is easy to show by simple integration by parts that, if a function $x \in K(\downarrow, \uparrow)$ has absolutely continuous first derivative, then

$$
\begin{gathered}
x(t)=\int_{0}^{t}\left(\int_{s}^{\infty} z(\tau) d \tau\right) d s \\
=t \int_{t}^{\infty} z(s) d s+\int_{0}^{t} s z(s) d s=Q_{1} z(t)+P_{1} z(t),
\end{gathered}
$$

where $z(s)$ is a nonnegative function. We can set $z(s) \equiv$ $-x^{\prime \prime}(s)$.

Theorem 4. Suppose that $p \in(1, \infty)$ and $w$ is a weight function such that, for any $t \in R_{+}$,

$$
\int_{0}^{\infty}\left(\min \left\{1, \frac{s}{t}\right\}\right)^{p} w^{p}(s) d s<\infty .
$$

Consider the cone $K(\downarrow, \uparrow) \cap L_{w}^{p}$.

Let $w_{0}$ and $w_{1}$ be the new weight functions defined by the equalities

$$
\left\|\kappa(t, \infty) \frac{1}{w_{0}(s)}\left|L^{p^{\prime}}\|\cdot\| \kappa(0, t) s w(s)\right| L^{p}\right\| \equiv 1,
$$

and

$$
\left\|\kappa(0, t) \frac{s}{w_{1}(s)}\left|L^{p^{\prime}}\|\cdot\| \kappa(t, \infty) w(s)\right| L^{p}\right\| \equiv 1
$$

for all $t>0$, and let

$$
V(t)=\max \left\{w_{0}(t), w_{1}(t)\right\} .
$$

Then, the following assertions are valid: the sum of operators $Q_{1}+P_{1}$ acts and is bounded in the pair

$$
\left(Q_{1}+P_{1}\right):\left(L_{\mathrm{v}}^{p}\right)_{+} \rightarrow K(\downarrow, \uparrow) \cap L_{w}^{p} ;
$$

for any $y \in K(\downarrow, \uparrow) \cap L_{w}^{p}$ with $\left\|y \mid L_{w}^{p}\right\|=1$, there exists a function $x \in L_{V}^{p}$ with $\left\|x \mid L_{V}^{p}\right\|=1$ such that

$$
\left(\left(Q_{1}+P_{1}\right) x\right)(t) \geq c y(t)
$$


for all $t \in(0, \infty)$, where $c>0$ is a constant, if and only if

$$
\begin{gathered}
\inf _{t}\left\{\left(\left\|\kappa(0, t) \frac{s}{V(s)}\left|L^{p^{\prime}}\|+t\| \kappa(t, \infty) \frac{s}{V(s)}\right| L^{p^{\prime}}\right\|\right)\right. \\
\left.\times\left(\left\|\kappa(0, t) \frac{s}{t} w(s)\left|L^{p}\|+\| \kappa(t, \infty) w(s)\right| L^{p}\right\|\right)\right\}<\infty .
\end{gathered}
$$

Condition (6) ensures that the extreme functions $\min \left\{1, \frac{s}{t}\right\}$ belong to the cone $K(\downarrow, \uparrow)$ of the space $L_{w}^{p}$. Condition (7) is necessary and sufficient for the boundedness of $Q_{1}$ as an operator from $L_{w_{0}}^{p}$ in $L_{w}^{p}$, and condition (8) is necessary and sufficient for the boundedness of $P_{1}$ as an operator from $L_{w_{1}}^{p}$ to $L_{w}^{p}$. Therefore, if $V$ is chosen as in the statement of the theorem, then condition (9) does hold.

Condition (11) in Theorem 4 simply ensures the possibility that condition (10) holds for the family of extreme functions $\min \left\{1, \frac{s}{t}\right\}$ of the cone $K(\downarrow, \uparrow)$.

For a function $y \in K(\downarrow, \uparrow) \cap L_{w}^{p}$, the function $x$ in (10) is defined constructively.

Theorem 4 has an analogue for the cones $K(\varphi, \psi)=$ $\left\{x: R_{+} \rightarrow R_{+}: x(t) \cdot \varphi\right.$ is nondecreasing and $\psi(t) \cdot x(t)$ is nonincreasing .

Condition (11) does not always hold. There are various sufficient conditions under which (11) holds. In particular, on the power scale, i.e., for $w(t)=t^{\alpha}$, conditions (11) are satisfied for $\alpha \in\left(-\frac{1}{p}-1,-\frac{1}{p}\right)$.

The following theorem exemplifies the applications of Theorem 4.

Theorem 5. Suppose that $p \in(1, \infty)$ and $w$ is a weight function satisfying (6). Let $w_{0}, w_{1}$, and $v$ be functions such that condition (11) holds for this $w$, and let $Y$ be an ideal Banach space in $S(\mu)$.

A sublinear operator $T$ acts and is bounded as an operator from $K(\downarrow, \uparrow) \cap L_{w}^{p}$ to $Y$ if and only if the superposition operator $T\left(Q_{1}+P_{1}\right)$ acts and is bounded as an operator from $L_{\mathrm{V}}^{p}$ to $Y$.

Now, we proceed to extrapolation theorems for operators on cones. We need some additional constructions.

Let $X_{0}$ and $X_{1}$ be two ideal spaces in $S(\mu)$.
Take $0<\theta<1$. Consider the new ideal space $X_{0}^{\theta} X_{1}^{1-\theta}$ (the Calderon-Lozanovskii construction) consisting of those $x \in S(\mu)$ for which the norm

$$
\begin{gathered}
\|x\|_{X_{0}^{\theta} X_{1}^{1-\theta}}=\inf \left\{\lambda>0:|x(t)| \leq \lambda \cdot\left|x_{0}(t)\right|^{\theta}\left|x_{1}(t)\right|^{1-\theta}\right. \\
\left.\quad \text { for any } t \in \Omega ; \quad\left\|x_{0}\right\|_{X_{0}} \leq 1, \quad\left\|x_{1}\right\|_{X_{1}} \leq 1\right\}
\end{gathered}
$$

is finite. The space $X_{0}^{\theta} X_{1}^{1-\theta}$ was introduced by Calderon [13] in studying the complex interpolation method.

If $K$ is a cone in $S(\mu)$, then we can define a new cone $\left(K \cap X_{0}\right)^{\theta}\left(K \cap X_{1}\right)^{1-\theta}$, by analogy with $X_{0}^{\theta} X_{1}^{1-\theta}$, namely, by taking only decompositions into elements of the cone in (12). The following theorem is an interpolation result; it is well known for the cone of nonnegative functions (see, e.g., $[14,15]$ ).

Theorem 6. Suppose that $T$ is a positive operator and $K_{0}$ and $K_{1}$ are two cones in $S(\mu)_{+}$. Let $X_{0}, X_{1}, Y_{0}$, and $Y_{1}$ be ideal Banach spaces in $S(\mu)$. Suppose that an operator T acts and is bounded as an operator T: $X_{i} \cap$ $K_{0} \rightarrow Y_{i} \cap K_{1}$ for $i=0,1$. Let $\theta \in(0,1)$.

Then, the operator $T$ acts and is bounded as an operator T: $\left(K_{0} \cap X_{0}\right)^{\theta}\left(K_{0} \cap X_{1}\right)^{1-\theta} \rightarrow\left(K_{1} \cap Y_{0}\right)^{\theta}\left(K_{1} \cap\right.$ $\left.Y_{1}\right)^{1-\theta}$.

Remark 2. As is usual in interpolation theory, for an arbitrary cone $K$, the equality $\left(K \cap L_{\mathrm{v}_{0}}^{1}\right)^{\theta}\left(K \cap L_{\mathrm{v}_{1}}^{\infty}\right)^{1-\theta}=$ $K \cap\left(\left(L_{V_{0}}^{1}\right)^{\theta}\left(L_{V_{1}}^{\infty}\right)^{1-\theta}\right)$ does not always hold, even for the cone $K(\downarrow)$.

Theorem 7. Suppose that $p \in(1, \infty), w$ is a weight function satisfying conditions (1) and (2), and $v$ is $a$ function constructed for $w$ according to (3). Let $\theta=\frac{1}{p}$. Suppose that $T$ is a linear positive operator $T$ acting and bounded in the pair

$$
T: K(\downarrow) \cap L_{w}^{p} \rightarrow L_{w}^{p} .
$$

Then, there exist functions $v_{0}, v_{1}, u_{0}$, and $u_{1}$ such that

$$
v_{0}^{\theta}(t) \cdot v_{1}^{1-\theta}(t) \equiv v(t), \quad u_{0}^{\theta}(t) \cdot u_{1}^{1-\theta}(t) \equiv u(t) ;
$$

the operator $T Q$ acts and is bounded in the pairs

$$
T Q: L_{v_{0}}^{1} \rightarrow L_{u_{0}}^{1}, \quad T Q: L_{v_{1}}^{\infty} \rightarrow L_{u_{1}}^{\infty} .
$$

Combining Theorems 6 and 7, we obtain the following extrapolation theorem for operators on the cone $K(\downarrow)$.

Theorem 8. Suppose that $p \in(1, \infty) ; w$ is a weight function satisfying condition (6); $w_{0}, w_{1}$, and $v$ are functions constructed for $w$; and condition (11) holds. Let $\theta=\frac{1}{p}$. Suppose that $T$ is a linear positive operator acting and bounded in the pair

$$
T: K(\downarrow, \uparrow) \cap L_{w}^{p} \rightarrow L_{u}^{p} .
$$


Then, there exist functions $v_{0}, v_{1}, u_{0}$, and $u_{1}$ such that relations (13) hold and the operator $T\left(Q_{1}+P_{1}\right)$ acts and is bounded in the pairs

$$
T\left(Q_{1}+P_{1}\right): L_{\mathrm{v}_{0}}^{1} \rightarrow L_{u_{0}}^{1}, \quad T\left(Q_{1}+P_{1}\right): L_{\mathrm{v}_{1}}^{\infty} \rightarrow L_{u_{1}}^{v} .
$$

Combining Theorems 6 and 7, we obtain an extrapolation theorem for operators on the cone $K(\downarrow, \uparrow)$.

\section{ACKNOWLEDGMENTS}

This work was supported by the grant of the Swedish Royal Academy of Sciences for collaboration with Russia (project no. 35160). E.I. Berezhnoi acknowledges the support of the Russian Foundation for Basic Research (project no. 05-01-00206).

\section{REFERENCES}

1. E. T. Sawyer, Stud. Math. 96, 145-158 (1990).

2. E. I. Berezhnoi, Tr. Mat. Inst. im. V.A. Steklova, Ross. Akad. Nauk 204, 3-36 (1993).

3. H. Heinig and L. Maligranda, Stud. Math. 116, 133-165 (1995).
4. A. Kufner and L.-E. Persson, Weighted Inequalities of Hardy Type (World Sci., Singapore, 2003).

5. V. B. Korotkov, Integral Operators (Nauka, Novosibirsk, 1983) [in Russian].

6. J. Garcia-Cuerva and J. Rubio De Francia, Weighted Norm Inequalities and Related Topics (North-Holland, Amsterdam, 1985).

7. E. I. Berezhnoi, Dokl. Akad. Nauk 344, 727-730 (1995).

8. E. I. Berezhnoi and L. Maligranda, Dokl. Akad. Nauk 393, 583-586 (2003) [Dokl. Math. 68, 392-395 (2003)].

9. S. G. Krein, Yu. I. Petunin, and E. M. Semenov, Interpolation of Linear Operators (Nauka, Moscow, 1978) [in Russian].

10. V. G. Maz'ja, Sobolev Spaces (Springer-Verlag, Berlin, 1985).

11. E. I. Berezhnoi, Tr. Mat. Inst. im. V.A. Steklova, Akad. Nauk SSSR 201, 26-42 (1991).

12. E. I. Berezhnoi, Proc. Am. Math. Soc. 127 (1), 79-87 (1999).

13. A. P. Calderon, Matematika 9 (3), 56-129 (1965).

14. E. I. Berezhnoi, in Qualitative and Approximate Methods for the Study of Operator Equations (Yaroslav. Gos. Univ., Yaroslavl, 1981), pp. 3-12 [in Russian].

15. L. Maligranda, Orlicz Spaces and Interpolation (Campinas, 1989). 